\title{
NUMERICAL STUDY OF THERMOPHORESIS ON AEROSOL PARTICLE DEPOSITION FROM HIEMENZ FLOW THROUGH POROUS MEDIUM ONTO A STRETCHING SURFACE
}

\author{
Jian-Sheng Huang \\ Department of Architecture, National Taiwan University of Science and Technology, Taipei, Taiwan, R.O.C, \\ lilysonq@yahoo.com.tw \\ Ruey-Yih Tsai \\ Department of Mechanical Engineering, Chung Yuan Christian University, Chung Li, Taiwan, R.O.C \\ Kuei-Hsun Huang \\ Department of Living Services Industry, Chin Min Institute of Technology, Toufen, Taiwan, R.O.C
}

Follow this and additional works at: https://jmstt.ntou.edu.tw/journal

Part of the Other Civil and Environmental Engineering Commons

\footnotetext{
Recommended Citation

Huang, Jian-Sheng; Tsai, Ruey-Yih; and Huang, Kuei-Hsun (2012) "NUMERICAL STUDY OF THERMOPHORESIS ON AEROSOL PARTICLE DEPOSITION FROM HIEMENZ FLOW THROUGH POROUS MEDIUM ONTO A STRETCHING SURFACE," Journal of Marine Science and Technology. Vol. 20: Iss. 2, Article 7. DOI: $10.51400 / 2709-6998.1835$

Available at: https://jmstt.ntou.edu.tw/journal/vol20/iss2/7

This Research Article is brought to you for free and open access by Journal of Marine Science and Technology. It has been accepted for inclusion in Journal of Marine Science and Technology by an authorized editor of Journal of Marine Science and Technology.
} 
NUMERICAL STUDY OF THERMOPHORESIS ON AEROSOL PARTICLE DEPOSITION FROM HIEMENZ FLOW THROUGH POROUS MEDIUM ONTO A STRETCHING SURFACE

\section{Acknowledgements}

The authors are grateful to the National Science Council, Taiwan, ROC for support through grant No. NSC 98-2221-E033-055 


\title{
NUMERICAL STUDY OF THERMOPHORESIS ON AEROSOL PARTICLE DEPOSITION FROM HIEMENZ FLOW THROUGH POROUS MEDIUM ONTO A STRETCHING SURFACE
}

\author{
Jian-Sheng Huang ${ }^{1}$, Ruey-Yih Tsai ${ }^{2}$, and Kuei-Hsun Huang ${ }^{3}$
}

Key words: Hiemenz flow, porous medium, thermophoresis, particle deposition, stretching surface.

\begin{abstract}
A theoretical study of aerosol particles responding to thermophoresis, involving the particle deposition rate onto a stretching permeable surface with internal heat source is proposed. The effects on particle transport mechanisms include Brownian diffusion, thermophoresis, porosity, stretching surface, heat source, and suction/injection velocity. The governing equations of continuity, momentum, energy, and particle concentration are transformed using similarity analysis, and the solutions are obtained through appropriate numerical schemes. The predicted results show that if the thermophoretic and stretching parameters increase, the deposition velocity increases for a cold surface. However, the deposition velocity decreases rapidly for a hot surface as the thermophoretic parameter increases. By way of the modeling analysis, the particle deposition velocities are calculated to control the particle mobility from the air.
\end{abstract}

\section{INTRODUCTION}

Aerosol particle is a kind of airborne pollutant source, and the deposition mechanism due to thermophoresis is important in many practical issues. Thermophoresis is a radiometric force by temperature gradient that enhances small particles moving toward a cold surface and away from a hot one. It plays a significant role on particle transport in laminar boundary layer flow. Generally, the mainly effect of thermophoresis

Paper submitted 06/25/10; revised 09/21/10; accepted 10/12/10. Author for correspondence: Jian-Sheng Huang (e-mail: lilysonq@yahoo.com.tw).

${ }^{I}$ Department of Architecture, National Taiwan University of Science and Technology, Taipei, Taiwan, R.O.C.

${ }^{2}$ Department of Mechanical Engineering, Chung Yuan Christian University, Chung Li, Taiwan, R.O.C.

${ }^{3}$ Department of Living Services Industry, Chin Min Institute of Technology, Toufen, Taiwan, R.O.C. on small particle size is especially effective in a range of $d p=0.01-1.0 \mu \mathrm{m}$. Particle deposition from a moving air stream onto a surface caused by thermophoresis is widely seen in a lot of engineering applications, such as particle deposition onto a wafer surface in the modern semiconductor industry, electronic component cooling using a fan, filtration process in gas-cleaning, problems for nuclear reactor safety, clean room and human healthy topics, etc. It has also been proved that thermophoresis is the dominant mass transport mechanism in the chemical vapor deposition process used in the fabrication of optical fibers. Commonly, the deposition mechanisms for particles include Brownian diffusion, convection, thermophoresis and other mechanisms, e.g. electrophoresis [31].

Thermophoresis on particle deposition onto a surface in laminar boundary layer flow is now rather well understood theoretically. Goren [12] developed the thermophoretic deposition of particles in a laminar compressible boundary layer flow past a flat plate. There are some other proposed models for particle deposition by coupled of thermophoresis and Brownian diffusion (Homsy et al. [15]; Batchelor and Shen [5]). Peters and Cooper [21], Opiolka et al. [19], and Tsai [30] dealt with the coupled of thermophoresis, forced convection and other effects on the predicted deposition rates for a stagnation point flow. Nazaroff and Cass [18] calculated the particle deposition rates due to combined effects of thermophoresis and natural convection. Chang et al. [8] proposed a theoretical study on the effect of thermophoresis for aerosol particle deposition from a mixed convection flow onto a vertical flat plate. Selim et al. [27] discussed the effect of surface mass transfer on mixed convection flow past a heated vertical permeable plate with thermophoresis. Chamkha et al. [6, 7] studied the effect of thermophoretic force in free convection boundary layer from a vertical flat plate embedded in a porous medium with heat generation or absorption. Seddeek [26] analyzed mixed convection flow, heat, and mass transfer about an isothermal vertical flat plate embedded in a fluid-saturated porous medium while the effects of viscous dissipation and thermophoresis in both aiding and opposing flows are considered. Postelnicu [22] looked into the thermophoresis particle deposition effect on the free convection over a horizontal 
flat plate embedded in a fluid-saturated porous medium. Alam et al. [1] presented a two-dimensional steady MHD mixed convection and mass transfer flow over a semi-infinite porous inclined plate in the presence of thermal radiation with variable suction and thermophoresis numerically. Rashad [23] focused on the research of magnetohydrodynamic and thermal radiation effects on heat and mass transfer in steady laminar boundary layer flow of a Newtonian, viscous fluid over a vertical flat plate embedded in a fluid saturated porous medium in the presence of thermophoretic particle deposition effect. Partha [20] used similarity technique to obtain the solutions about effect of suction/injection on thermophoretic particle deposition in free convection onto a vertical plate embedded in a fluid saturated non-Darcy porous medium. Mahdy and Hady [16] pointed out the effects of thermophoretic particle deposition on the free convective flow over a vertical flat plate embedded in a non-Newtonian fluid saturated porous medium in the presence of a magnetic field.

Plane stagnation point flow was first studied by Hiemenz [13] who analyzed the Navier-Stokes equation to obtain the flow field solution and called as Hiemenz flow. Subsequently, the flow and heat transfer phenomena related to Hiemenz flow problem was proposed by Goldstein [11]. Three-dimensional axisymmetric stagnation point flow was reported by Homann [14] while the Navier-Stokes equation was transformed into a third-order ordinary differential equation using similarity technique. The heat transfer condition and temperature distribution of stagnation point flow were analyzed by Sibulkin [28]. Sakiadis [25] initiated the study of boundary layer flow over a continuous solid surface moving with a constant speed. The flow field of stretching surface with a power-law variation velocity was discussed by Banks [4] and Ali [2]. Elbashbeshy and Bazid [9, 10] considered the flow over a porous medium onto a stretching surface for different permeability and injection parameters. Attia [3] investigated into the conducting fluid impinging on a permeable stretching surface with heat generation while flow through a porous medium. Tsai and Huang [32] studied the heat and mass transfer for Soret and Dufour's effects on Hiemenz flow through porous medium onto a stretching surface.

So far, there were still relative few published papers focused on the rate of thermophoretic deposition for different particle sizes onto a moving permeable surface in a flow system through a porous medium with temperature gradient. In this work, we are interested in the deposition problem from a higher temperature air-particle flow onto an adjacent cold surface or from a lower temperature air-particle flow onto a hot one. The particle deposition velocities are calculated to determine the interactive effects from the physical model.

\section{MATHEMATICAL FORMULATION}

The air-particle flow is modeled as a two-dimensional, incompressible and steady state laminar flow near a stagnation point at a stretching surface coinciding with the plate at $y=0$,

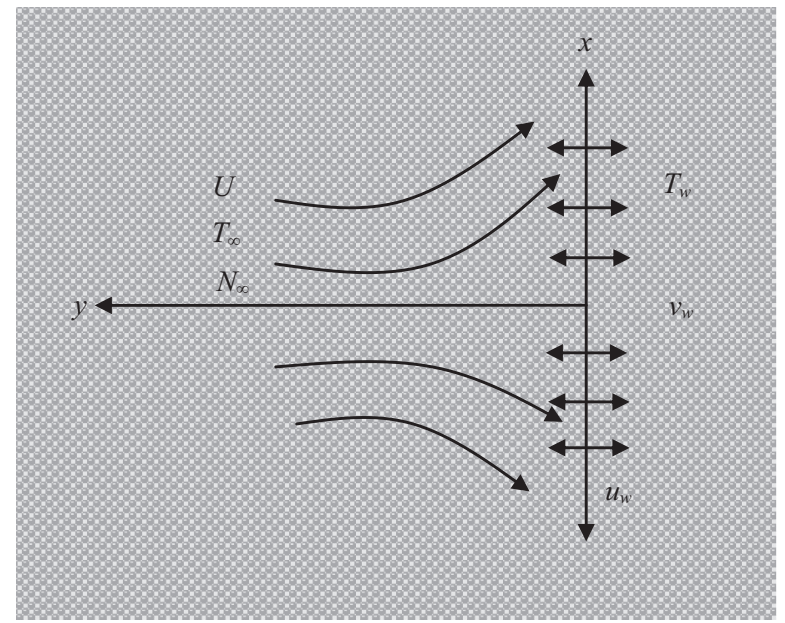

Fig. 1. Schematic of Hiemenz flow through a porous medium physical model and coordinate system.

the flow being in a region $y>0$ is shown in Fig. 1. Two equal and opposite forces are introduced along the $x$-axis the surface. The flow is considered from the $y$-axis to impinge onto the flat surface. The surface is considered as a permeable wall with suction or injection flow through it and the Darcy's model can be applied. The flow condition at $y \rightarrow \infty$ is given by $U(x)=a x$ and $V(y)=-a y$, where $a$ denotes the free stream strength. The temperature at the wall surface maintains a constant, $T_{w}$ and the free stream is at another temperature, $T_{\infty}$.

The energy transport is considered with the heat generation/absorption effect. The concentration of particles is assumed to be dilute limit where the presences of the particles do not affect the host flow velocity. The governing equations based on the conservation of mass, momentum, energy, and particle transport using the boundary layer approximation are [33]

$$
\begin{gathered}
\frac{\partial u}{\partial x}+\frac{\partial v}{\partial y}=0 \\
\rho\left(u \frac{\partial u}{\partial x}+v \frac{\partial u}{\partial y}\right)=\rho U \frac{d U}{d x}+\mu\left(\frac{\partial^{2} u}{\partial y^{2}}\right)+\frac{\mu}{K}(U-u) \\
\rho c_{p}\left(u \frac{\partial T}{\partial x}+v \frac{\partial T}{\partial y}\right)=k \frac{\partial^{2} T}{\partial y^{2}}+Q\left(T-T_{\infty}\right) \\
u \frac{\partial N}{\partial x}+v \frac{\partial N}{\partial y}+\frac{\partial}{\partial y}\left(N V_{T}\right)=D \frac{\partial^{2} N}{\partial y^{2}}
\end{gathered}
$$

where $\rho, \mu, k$ and $c_{p}$ are the density, dynamic viscosity, thermal conductivity and the specific heat at constant pressure, separately. $K$ is the Darcy permeability, $Q$ is the volumetric heat generation/absorption rate, and $N$ is the particle concentration. $V_{T}$ is called as thermophoretic velocity recommended by Talbot et al. [29] 


$$
V_{T}=-\kappa \frac{v}{T} \frac{\partial T}{\partial y}
$$

and the value of $\kappa v$ represents the thermophoretic diffusivity, and $\kappa$ is the thermophoretic coefficient defined using

$$
\kappa=\frac{2 C_{s}\left(\lambda_{g} / \lambda_{p}+C_{t} K n\right) C_{c}}{\left(1+3 C_{m} K n\right)\left(1+2 \lambda_{g} / \lambda_{p}+2 C_{t} K n\right)}
$$

where $\lambda_{g}$ and $\lambda_{p}$ are the thermal conductivities of air and particle, respectively. $C_{s}=1.147, C_{t}=2.20$, and $C_{m}=1.146$ are constants obtained from the experimental data. $C_{c}=1+$ $K n\left(C_{1}+C_{2} \exp \left(-C_{3} / K n\right)\right)$ is the Stokes-Cunningham correction factor and $K n\left(=2 \lambda / d_{p}\right)$ is Knudsen number, where $C_{1}=$ $1.2, C_{2}=0.41, C_{3}=0.88$, and $\lambda$ is the mean free path of air molecule [5].

The boundary conditions at $y=0$ and $y \rightarrow \infty$ are

$$
\begin{gathered}
y=0 ; \quad u=u_{w}=c x, \quad v=v_{w}, \quad T=T_{w}, \quad N=0 \\
y \rightarrow \infty ; \quad u \rightarrow U=a x, \quad T=T_{\infty}, \quad N=N_{\infty}
\end{gathered}
$$

where $c$ is a positive constant that represents the characteristic stretching intensity, and $v_{w}$ means the suction/injection velocity at the permeable surface.

We transform the governing partial differential Eqs. (1)-(4) into (8)-(10) by similarity techniques. Generally speaking, a similarity solution is one in which the number of variables can be reduced by one or more by some analytical means, usually by a coordinate transformation. The benefits of similarity analysis are immense; the problem in this paper reduces a set of nonlinear partial differential equations into ordinary differential equations, which we can handle with a numerical method such as Runge-Kutta integration [33]. The governing partial differential Eqs. (1)-(4) admit similarity solutions for obtaining the dimensionless stream function $f(\eta)$, temperature $\theta(\eta)$, and particle concentration $\phi(\eta)$. The dimensionless parameters are introduced as

$$
\begin{aligned}
& \psi=\sqrt{c v} x f(\eta), u=c x f^{\prime}(\eta), v=-\sqrt{c v} f(\eta), \eta=\sqrt{\frac{c}{v}} y, \\
& \theta(\eta)=\frac{T_{\infty}-T}{T_{\infty}-T_{w}}, \phi(\eta)=\frac{N}{N_{\infty}}
\end{aligned}
$$

where $v(=\mu l \rho)$ is the kinematic viscosity for air $(v=1.15 \times$ $10^{-5} \mathrm{~m}^{2} / \mathrm{s}$ used). Using the similarity techniques, Eqs. (1)-(4) can be transformed into a following forms in terms with $f(\eta)$, $\theta(\eta)$, and $\phi(\eta)$ expressed as

$$
\begin{gathered}
f^{\prime \prime \prime}+f f^{\prime \prime}-\left(f^{\prime}\right)^{2}+M\left(C-f^{\prime}\right)+C^{2}=0 \\
\theta^{\prime \prime}+\operatorname{Pr} f \theta^{\prime}+\operatorname{Pr} B \theta=0 \\
\frac{1}{S c} \phi^{\prime \prime}+\left(f-N_{t} \theta^{\prime}\right) \phi^{\prime}-\left[N_{t} \theta^{\prime \prime}+\frac{N_{t}^{2}}{\kappa}\left(\theta^{\prime}\right)^{2}\right] \phi=0
\end{gathered}
$$

and the boundary conditions become

$$
\begin{array}{r}
\eta=0 ; \quad f(0)=f_{w}, \quad f^{\prime}(0)=1, \quad \theta(0)=1, \quad \phi(0)=0 \\
\eta \rightarrow \eta_{\infty} ; \quad f^{\prime}(\infty)=C, \quad \theta(\infty)=0, \quad \phi(\infty)=1
\end{array}
$$

where $M(=v / c K)$ is the porosity parameter and $C(=a / c)$ is the stretching parameter which stands for the ratio of free stream strength to surface stretching intensity. $f_{w}\left(=v_{w} / \sqrt{c v}\right)$ is the wall suction/injection parameter, for surface injection, coincides with $f_{w}<0$, but for surface suction $f_{w}>0 . \operatorname{Pr}(=v / \alpha)$ is Prandtl number for the air $(\operatorname{Pr}=0.7$ used $), S c(=v / D)$ is particle Schmidt number and the selected values ranges from 2.90 to 6.37E+07 corresponding to the particle diameters from $d_{p}=$ 0.001-100 $\mu \mathrm{m}$, and $B\left(=Q / c \rho c_{p}\right)$ is the heat source parameter. $N_{t}(=-\kappa \Delta T / T)$ represents the thermophoretic parameter by taking $\Delta T / T \approx\left(T_{w}-T_{\infty}\right) / T_{\infty}$ if the temperature difference is small compared with the ambient temperature, $T_{\infty}=300 \mathrm{~K}$ assumed at one atmospheric pressure. For cold surface condition responds to $N_{t}>0$, whereas for hot one $N_{t}<0$.

Usually, the shear stress and heat flux on the surface for the flow and temperature fields are of most interest in such a problem and measure the dimensionless quantities, $f^{\prime \prime}(0)$ and $\theta^{\prime}(0)$, respectively. In this study, we examine the particle transport due to the thermophoretic effect from the temperature gradient. Using Fick's law, in the mass transfer analysis, the particle flux is given by the definition [17]

$$
J=-D \frac{\partial N}{\partial y}+\left(v+V_{T}\right) N
$$

that leads to Eq. (12), at $y=0$, by taking into account that here $N=0$, see Eq. (7a), and the deposition flux at the wall surface is

$$
J_{w}=-\left.D \frac{\partial N}{\partial y}\right|_{y=0}=-D \phi^{\prime}(0) N_{\infty} \sqrt{\frac{c}{v}}=-\frac{1}{S c} \phi^{\prime}(0) N_{\infty} \sqrt{c v}
$$

However, the engineer is usually more interested in the particle deposition velocity, $V_{d}$ which is customarily defined as deposition flux divided by the free stream concentration, $N_{\infty}$

$$
V_{d}=\frac{J_{w}}{N_{\infty}}=-\frac{1}{S c} \phi^{\prime}(0) \sqrt{c v}
$$

where $\phi^{\prime}(0)$ denotes the slope of concentration profile at the wall.

\section{RESULTS AND DISCUSSION}

In order to gain the physical insight, Eqs. (8)-(10) constitute a nonlinear boundary value problem. The obtained governing Eqs. (8)-(10), with the associated boundary conditions (11), are solved numerically using the fourth order Runge-Kutta integration algorithm with a systematic estimate of $f^{\prime \prime}(0), \theta^{\prime}(0)$ 


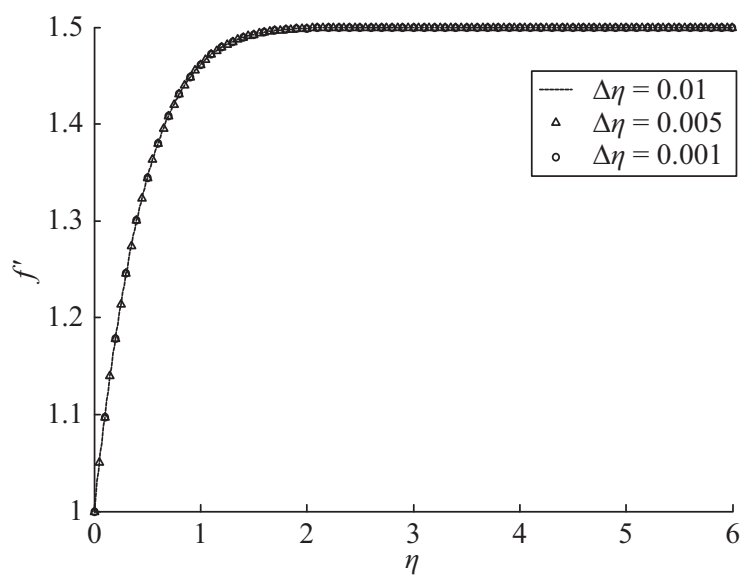

Fig. 2. Velocity profiles for different step sizes $\Delta \eta$.

and $\phi^{\prime}(0)$ by Newton-Raphson shooting technique. Based on the Runge-Kutta scheme, the higher-order differential equations can easily be converted to a system of first-order differential equations by introducing extra variables. The fourth order Runge-Kutta method is a most popular and good choice for common purposes because it is quite accurate, stable, and easy to program. Most authorities proclaim that it is not necessary to go to a higher-order method because the increased accuracy is offset by additional computational effort. If more accuracy is required, a smaller step size should be used. In order to verify the effects of step size $\Delta \eta$, we run the code for our model with three different step sizes as $\Delta \eta=0.01, \Delta \eta=$ $0.005, \Delta \eta=0.001$ and in each case we found excellent agreement among them [1]. Fig. 2 shows the velocity profiles for different step sizes.

The step size $\Delta \eta=0.001$ is used to obtain the numerical solution for the following cases and the boundary condition $\eta \rightarrow \infty$ is approximated by $\eta_{\max }=6.0$, which is sufficiently large for the velocity to approach the relevant stream velocity. The values of $f$ and $\theta$ are firstly solved by way of numerical calculation for Eqs. (8) and (9), after that the particle concentration $\phi$ will be obtained. Criterion for convergence rates used for the ratio of $f^{\prime}, \theta$, and $\phi$ at the last two approximations are less than $10^{-6}$ at all $\eta$ in $0<\eta<\eta_{\infty}$ values.

$$
\begin{aligned}
& \frac{\left|f_{n+1}^{\prime}-f_{n}^{\prime}\right|}{f_{n+1}^{\prime}} \leq 10^{-6} \\
& \frac{\left|\theta_{n+1}-\theta_{n}\right|}{\theta_{n+1}} \leq 10^{-6} \\
& \frac{\left|\phi_{n+1}-\phi_{n}\right|}{\phi_{n+1}} \leq 10^{-6}
\end{aligned}
$$

In addition, for further validating the accuracy of our numerical method, we have compared our results with the previous published data from textbook [33] in Table 1, and the comparisons in all cases are found to be in good agreement.

\begin{tabular}{|c|c|c|}
\hline \multicolumn{3}{|c|}{$f^{\prime \prime}(0)$} \\
\hline & White [33] & Present results \\
\hline & 1.23259 & 1.2325 \\
\hline \multicolumn{3}{|c|}{$\theta^{\prime}(0)$} \\
\hline $\operatorname{Pr}$ & White [33] & Present results \\
\hline 0.1 & 0.220 & 0.2201 \\
\hline 1.0 & 0.570 & 0.5704 \\
\hline 10 & 1.339 & 1.3385 \\
\hline 100 & 2.986 & 2.9855 \\
\hline 1000 & 6.529 & 6.5288 \\
\hline
\end{tabular}

Table 1. Comparison values of $f^{\prime \prime}(0)$ and $\theta^{\prime}(0)$ at $M=0.0$, $C=\mathbf{1 . 0}$ and $B=0.0$.

Table 2. The prediction values of $f^{\prime \prime}(0)$ at different $C$ and $M$ for $f_{w}=0.1$.

\begin{tabular}{cccc}
\hline \multicolumn{4}{c}{$f^{\prime \prime}(0), f_{w}=0.1$} \\
\hline$M$ & $C=0.5$ & $C=1.0$ & $C=1.5$ \\
0 & -0.6944 & 0 & 0.9374 \\
1.0 & -0.8586 & 0 & 1.0638 \\
2.0 & -0.9963 & 0 & 1.1768 \\
3.0 & -1.1170 & 0 & 1.2800 \\
\hline
\end{tabular}

Table 3. The prediction values of $\theta^{\prime}(0)$ at different $C, M$ and $B$ for $f_{w}=0.1, \operatorname{Pr}=0.7$.

\begin{tabular}{ccccccc}
\hline \multicolumn{4}{c}{$\theta^{\prime}(0), f_{w}=0.1, B=0.1$} & \multicolumn{3}{c}{$\theta^{\prime}(0), f_{w}=0.1, B=-0.1$} \\
\hline$M$ & $C=0.5$ & $C=1.0$ & $C=1.5$ & $C=0.5$ & $C=1.0$ & $C=1.5$ \\
0 & -0.5554 & -0.6662 & -0.7591 & -0.6684 & -0.7572 & -0.8375 \\
1.0 & -0.5439 & -0.6662 & -0.7632 & -0.6590 & -0.7572 & -0.8412 \\
2.0 & -0.5358 & -0.6662 & -0.7664 & -0.6524 & -0.7572 & -0.8442 \\
3.0 & -0.5297 & -0.6662 & -0.7691 & -0.6473 & -0.7572 & -0.8467 \\
\hline \multicolumn{4}{c}{$\theta^{\prime}(0), f_{w}=0.1, B=0.5$} & $\theta^{\prime}(0), f_{w}=0.1, B=-0.5$ \\
\hline$M$ & $C=0.5$ & $C=1.0$ & $C=1.5$ & $C=0.5$ & $C=1.0$ & $C=1.5$ \\
0 & -0.2497 & -0.4515 & -0.5833 & -0.8549 & -0.9174 & -0.9799 \\
1.0 & -0.2285 & -0.4515 & -0.5883 & -0.8480 & -0.9174 & -0.9831 \\
2.0 & -0.2136 & -0.4515 & -0.5923 & -0.8430 & -0.9174 & -0.9857 \\
3.0 & -0.2025 & -0.4515 & -0.5956 & -0.8392 & -0.9174 & -0.9878 \\
\hline
\end{tabular}

The resulting of $f^{\prime \prime}(0), \theta^{\prime}(0)$, and $\phi^{\prime}(0)$ values can represent the magnitude for the wall shear stress, heat flux and particle deposition flux, individually. Eq. (14) indicates that the deposition velocity, $V_{d}$ depends upon the slope of particle concentration profile $\phi^{\prime}(0)$ at the wall. Table 2 shows the calculated values of $f^{\prime \prime}(0)$ at different $M$ and $C$ under a suction flow $f_{w}=0.1$. As $C=1.0$, the wall shear stress approaches to zero due to the equivalent free stream strength compared to the stretching intensity while the reversal sign is seen between $C>1.0$ and $C<1.0$. The values of $\left|f^{\prime \prime}(0)\right|$ increase with the increasing $M$. That means the gradually increasing porosity parameters tend to enhance the values of wall shear stress, and the direction is opposite for $C=0.5$ and $C=1.5$. The predicted values of $-\theta^{\prime}(0)$ at different $M, C$, and $B$ with $f_{w}=$ 0.1 are captured in Table 3. With the same trend of the data, 
Table 4. The prediction values of $\phi^{\prime}(0)$ at different $C, M$ and $B$ for $f_{w}=0.1, \operatorname{Pr}=0.7, N_{t}=-0.05$ and $d p=1.0 \mu m$.

\begin{tabular}{cccc}
\hline \multicolumn{3}{c}{$\phi^{\prime}(0), f_{w}=0.1, B=0.1$} \\
\hline$M$ & $C=0.5$ & $C=1.0$ & $C=1.5$ \\
0.0 & $3.9458 \mathrm{E}+04$ & $3.6432 \mathrm{E}+04$ & $3.3894 \mathrm{E}+04$ \\
1.0 & $3.9772 \mathrm{E}+04$ & $3.6432 \mathrm{E}+04$ & $3.3783 \mathrm{E}+04$ \\
2.0 & $3.9993 \mathrm{E}+04$ & $3.6432 \mathrm{E}+04$ & $3.3694 \mathrm{E}+04$ \\
3.0 & $4.0161 \mathrm{E}+04$ & $3.6432 \mathrm{E}+04$ & $3.3619 \mathrm{E}+04$ \\
\hline \multicolumn{4}{c}{$\phi^{\prime}(0), f_{w}=0.1, B=0.5$} \\
\hline$M$ & $C=0.5$ & $C=1.0$ & $C=1.5$ \\
0.0 & $4.7735 \mathrm{E}+04$ & $4.2224 \mathrm{E}+04$ & $3.8626 \mathrm{E}+04$ \\
1.0 & $4.8314 \mathrm{E}+04$ & $4.2224 \mathrm{E}+04$ & $3.8489 \mathrm{E}+04$ \\
2.0 & $4.8719 \mathrm{E}+04$ & $4.2224 \mathrm{E}+04$ & $3.8380 \mathrm{E}+04$ \\
3.0 & $4.9024 \mathrm{E}+04$ & $4.2224 \mathrm{E}+04$ & $3.8289 \mathrm{E}+04$ \\
\hline \multicolumn{4}{c}{$\phi^{\prime}(0), f_{w}=0.1, B=-0.1$} \\
\hline$M$ & $C=0.5$ & $C=1.0$ \\
0.0 & $3.6406 \mathrm{E}+04$ & $3.3982 \mathrm{E}+04$ & $C=1.5$ \\
1.0 & $3.6663 \mathrm{E}+04$ & $3.3982 \mathrm{E}+04$ & $3.1788 \mathrm{E}+04$ \\
2.0 & $3.6844 \mathrm{E}+04$ & $3.3982 \mathrm{E}+04$ & $3.1686 \mathrm{E}+04$ \\
3.0 & $3.6983 \mathrm{E}+04$ & $3.3982 \mathrm{E}+04$ & $3.1536 \mathrm{E}+04$ \\
\hline \multicolumn{3}{c}{$\phi^{\prime}(0), f_{w}=0.1, B=-0.5$} \\
\hline$M$ & $C=0.5$ & $C=1.0$ \\
0.0 & $3.1380 \mathrm{E}+04$ & $2.9674 \mathrm{E}+04$ & $C=1.5$ \\
1.0 & $3.1570 \mathrm{E}+04$ & $2.9674 \mathrm{E}+04$ & $2.7977 \mathrm{E}+04$ \\
2.0 & $3.1706 \mathrm{E}+04$ & $2.9674 \mathrm{E}+04$ & $2.7807 \mathrm{E}+04$ \\
3.0 & $3.1811 \mathrm{E}+04$ & $2.9674 \mathrm{E}+04$ & $2.7748 \mathrm{E}+04$ \\
\hline \multicolumn{3}{c}{}
\end{tabular}

Table 5. The prediction values of $\phi^{\prime}(0)$ at different $C, M$ and $B$ for $f_{w}=0.1, \operatorname{Pr}=0.7, N_{t}=0.05$ and $d p=1.0 \mu m$.

\begin{tabular}{cccc}
\hline \multicolumn{3}{c}{$\phi^{\prime}(0), f_{w}=0.1, B=0.1$} \\
\hline$M$ & $C=0.5$ & $C=1.0$ & $C=1.5$ \\
0.0 & $6.9810 \mathrm{E}+04$ & $7.2829 \mathrm{E}+04$ & $7.5362 \mathrm{E}+04$ \\
1.0 & $6.9496 \mathrm{E}+04$ & $7.2829 \mathrm{E}+04$ & $7.5473 \mathrm{E}+04$ \\
2.0 & $6.9276 \mathrm{E}+04$ & $7.2829 \mathrm{E}+04$ & $7.5562 \mathrm{E}+04$ \\
3.0 & $6.9108 \mathrm{E}+04$ & $7.2829 \mathrm{E}+04$ & $7.5636 \mathrm{E}+04$ \\
\hline \multicolumn{3}{c}{$\phi^{\prime}(0), f_{w}=0.1, B=0.5$} \\
\hline$M$ & $C=0.5$ & $C=1.0$ & $C=1.5$ \\
0.0 & $6.1545 \mathrm{E}+04$ & $6.7050 \mathrm{E}+04$ & $7.0643 \mathrm{E}+04$ \\
1.0 & $6.0967 \mathrm{E}+04$ & $6.7050 \mathrm{E}+04$ & $7.0779 \mathrm{E}+04$ \\
2.0 & $6.0562 \mathrm{E}+04$ & $6.7050 \mathrm{E}+04$ & $7.0888 \mathrm{E}+04$ \\
3.0 & $6.0257 \mathrm{E}+04$ & $6.7050 \mathrm{E}+04$ & $7.0979 \mathrm{E}+04$ \\
\hline \multicolumn{3}{c}{$\phi^{\prime}(0), f_{w}=0.1, B=-0.1$} \\
\hline$M$ & $C=0.5$ & $C=1.0$ & $C=1.5$ \\
0.0 & $7.2854 \mathrm{E}+04$ & $7.5272 \mathrm{E}+04$ & $7.7461 \mathrm{E}+04$ \\
1.0 & $7.2598 \mathrm{E}+04$ & $7.5272 \mathrm{E}+04$ & $7.7562 \mathrm{E}+04$ \\
2.0 & $7.2417 \mathrm{E}+04$ & $7.5272 \mathrm{E}+04$ & $7.7644 \mathrm{E}+04$ \\
3.0 & $7.2279 \mathrm{E}+04$ & $7.5272 \mathrm{E}+04$ & $7.7712 \mathrm{E}+04$ \\
\hline \multicolumn{3}{c}{$\phi^{\prime}(0), f_{w}=0.1, B=-0.5$} \\
\hline$M$ & $C=0.5$ & $C=1.0$ \\
0.0 & $7.7864 \mathrm{E}+04$ & $7.9564 \mathrm{E}+04$ & $C=1.5$ \\
1.0 & $7.7674 \mathrm{E}+04$ & $7.9564 \mathrm{E}+04$ & $8.1356 \mathrm{E}+04$ \\
2.0 & $7.7539 \mathrm{E}+04$ & $7.9564 \mathrm{E}+04$ & $8.1426 \mathrm{E}+04$ \\
3.0 & $7.7434 \mathrm{E}+04$ & $7.9564 \mathrm{E}+04$ & $8.1484 \mathrm{E}+04$ \\
\hline \multicolumn{3}{c}{}
\end{tabular}

Table 6. The prediction values of $V_{d}(\mathrm{~m} / \mathrm{s})$ at different $M$ for $c=10, f_{w}=0.0, N_{t}=0.05, C=0.5$, and $B=0.0$.

\begin{tabular}{ccccc}
\hline$d p(\mu m)$ & $M=0.0$ & $M=1.0$ & $M=2.0$ & $M=3.0$ \\
\hline 0.001 & $5.346078 \mathrm{E}-03$ & $5.258511 \mathrm{E}-03$ & $5.192768 \mathrm{E}-03$ & $5.140314 \mathrm{E}-03$ \\
0.01 & $8.005400 \mathrm{E}-04$ & $8.005400 \mathrm{E}-04$ & $7.906766 \mathrm{E}-04$ & $7.874145 \mathrm{E}-04$ \\
0.1 & $3.675279 \mathrm{E}-04$ & $3.612086 \mathrm{E}-04$ & $3.567915 \mathrm{E}-04$ & $3.534578 \mathrm{E}-04$ \\
1.0 & $3.502790 \mathrm{E}-04$ & $3.436737 \mathrm{E}-04$ & $3.390510 \mathrm{E}-04$ & $3.355592 \mathrm{E}-04$ \\
10.0 & $3.495592 \mathrm{E}-04$ & $3.429402 \mathrm{E}-04$ & $3.383077 \mathrm{E}-04$ & $3.348082 \mathrm{E}-04$ \\
100.0 & $3.494969 \mathrm{E}-04$ & $3.428768 \mathrm{E}-04$ & $3.382433 \mathrm{E}-04$ & $3.347432 \mathrm{E}-04$ \\
\hline
\end{tabular}

Table 7. The prediction values of $V_{d}(\mathrm{~m} / \mathrm{s})$ at different $M$ for $c=10, f_{w}=0.0, N_{t}=0.05, C=1.5$, and $B=0.0$.

\begin{tabular}{ccccc}
\hline$d p(\mu m)$ & $M=0.1$ & $M=1.0$ & $M=2.0$ & $M=3.0$ \\
\hline 0.001 & $6.404481 \mathrm{E}-03$ & $6.441022 \mathrm{E}-03$ & $6.471051 \mathrm{E}-03$ & $6.496509 \mathrm{E}-03$ \\
0.01 & $8.889879 \mathrm{E}-04$ & $8.915055 \mathrm{E}-04$ & $8.935868 \mathrm{E}-04$ & $8.953629 \mathrm{E}-04$ \\
0.1 & $4.768264 \mathrm{E}-04$ & $4.792178 \mathrm{E}-04$ & $4.811338 \mathrm{E}-04$ & $4.827224 \mathrm{E}-04$ \\
1.0 & $4.633497 \mathrm{E}-04$ & $4.658032 \mathrm{E}-04$ & $4.677686 \mathrm{E}-04$ & $4.693975 \mathrm{E}-04$ \\
10.0 & $4.628038 \mathrm{E}-04$ & $4.652602 \mathrm{E}-04$ & $4.672277 \mathrm{E}-04$ & $4.688586 \mathrm{E}-04$ \\
100.0 & $4.627567 \mathrm{E}-04$ & $4.652133 \mathrm{E}-04$ & $4.671811 \mathrm{E}-04$ & $4.688121 \mathrm{E}-04$ \\
\hline
\end{tabular}

increasing $C$ increases the value of $-\theta^{\prime}(0)$ for both heat generative or absorptive conditions. For heat absorptive condition, $B<0$ is helpful for the heat transfer from the wall to the ambient. When the free stream strength is larger than the surface stretching intensity $C=1.5$ accompanies more significantly phenomena. Tables 4 and 5 show the predicted values of $\phi^{\prime}(0)$ at different $M, C$, and $B$ with $f_{w}=0.1, N_{t}= \pm 0.05$ and $d p=1.0$ $\mu m$. Totally speaking, the $\phi^{\prime}(0)$ becomes larger for a cold surface $N_{t}>0$, whereas smaller for a hot one. That implies the cold surface will enhance the particle deposition velocity. The relative parameters that affect the slope of concentration profiles can be analyzed from the displayed figures.

Tables 6-10 show the calculated particle deposition velocities at different particle sizes and parameters $C, M, f_{w}, B$ and $N_{t}$ for $c=10$. The particle deposition velocity varies with the porosity effect, $M$ and stretching effect, $C$ is revealed in Tables 6 and 7. The deposition velocity increases with increasing $C$ due to faster free stream strength. The larger particle size accompanies lower deposition velocity when airparticle flow through porous media with different permeability. Table 8 depicts the suction/injection effect at the permeable surface, the particle deposition velocity decreases for surface injection, $f_{w}<0$, but increases for surface suction, $f_{w}>0$ in the whole range of particle sizes $0.001 \leq d p \leq 100 \mu \mathrm{m}$. In Tables 9 and 10 , the deposition velocities are calculated with negative and positive $N_{t}$ for hot and cold surfaces, respectively. The deposition velocity increases with increasing $N_{t}$ for a cold surface whereas decreases for a hot one. This is because the effects of thermophoresis and convection are predicted to be particularly important for particles moving toward a cold surface or blowing away from a hot one at a given temperature gradient. 
Table 8. The prediction values of $V_{d}(\mathrm{~m} / \mathrm{s})$ at different $f_{w}$ for $c=10, N_{t}=0.05, M=1.0, c=1.0$, and $B=0.5$.

\begin{tabular}{cccccc}
\hline$d p(\mu m)$ & $f_{w}=-0.01$ & $f_{w}=0.0$ & $f_{w}=0.01$ & $f_{w}=0.05$ & $f_{w}=0.1$ \\
\hline 0.001 & $5.795494 \mathrm{E}-03$ & $5.874151 \mathrm{E}-03$ & $5.953254 \mathrm{E}-03$ & $6.274042 \mathrm{E}-03$ & $6.684559 \mathrm{E}-03$ \\
0.01 & $6.710490 \mathrm{E}-04$ & $7.572756 \mathrm{E}-04$ & $8.472328 \mathrm{E}-04$ & $1.236994 \mathrm{E}-03$ & $1.792941 \mathrm{E}-03$ \\
0.1 & $1.615341 \mathrm{E}-04$ & $2.715386 \mathrm{E}-04$ & $3.903741 \mathrm{E}-04$ & $8.852909 \mathrm{E}-04$ & $1.512459 \mathrm{E}-03$ \\
1.0 & $1.233512 \mathrm{E}-04$ & $2.484766 \mathrm{E}-04$ & $3.742114 \mathrm{E}-04$ & $8.780061 \mathrm{E}-04$ & $1.508174 \mathrm{E}-03$ \\
10.0 & $1.213212 \mathrm{E}-04$ & $2.474644 \mathrm{E}-04$ & $3.735405 \mathrm{E}-04$ & $8.777214 \mathrm{E}-04$ & $1.508008 \mathrm{E}-03$ \\
100.0 & $1.211399 \mathrm{E}-04$ & $2.473764 \mathrm{E}-04$ & $3.734825 \mathrm{E}-04$ & $8.776969 \mathrm{E}-04$ & $1.507994 \mathrm{E}-03$ \\
\hline
\end{tabular}

Table 9. The prediction values of $V_{d}(\mathrm{~m} / \mathrm{s})$ at different $N_{t}$ for $c=10, f_{w}=0.1, M=1.0, C=1.0$, and $B=0.5$.

\begin{tabular}{ccccc}
\hline$d p(\mu m)$ & $N_{t}=0.0$ & $N_{t}=-0.001$ & $N_{t}=-0.001$ & $N_{t}=-0.05$ \\
\hline 0.001 & $6.556966 \mathrm{E}-03$ & $6.554245 \mathrm{E}-03$ & $6.529457 \mathrm{E}-03$ & $6.412948 \mathrm{E}-03$ \\
0.01 & $1.529664 \mathrm{E}-03$ & $1.524401 \mathrm{E}-03$ & $1.477076 \mathrm{E}-03$ & $1.268424 \mathrm{E}-03$ \\
0.1 & $1.234343 \mathrm{E}-03$ & $1.228784 \mathrm{E}-03$ & $1.178755 \mathrm{E}-03$ & $9.566429 \mathrm{E}-04$ \\
1.0 & $1.229045 \mathrm{E}-03$ & $1.223461 \mathrm{E}-03$ & $1.173197 \mathrm{E}-03$ & $9.497438 \mathrm{E}-04$ \\
10.0 & $1.228840 \mathrm{E}-03$ & $1.223255 \mathrm{E}-03$ & $1.172982 \mathrm{E}-03$ & $9.494751 \mathrm{E}-04$ \\
100.0 & $1.228823 \mathrm{E}-03$ & $1.223237 \mathrm{E}-03$ & $1.172963 \mathrm{E}-03$ & $9.494519 \mathrm{E}-04$ \\
\hline
\end{tabular}

Table 10. The prediction values of $V_{d}(\mathrm{~m} / \mathrm{s})$ at different $N_{t}$ for $c=10, f_{w}=0.1, M=1.0, C=1.0$, and $B=0.5$.

\begin{tabular}{ccccc}
\hline$d p(\mu m)$ & $N_{t}=0.001$ & $N_{t}=0.01$ & $N_{t}=0.05$ & $N_{t}=0.1$ \\
\hline 0.001 & $6.559681 \mathrm{E}-03$ & $6.583818 \mathrm{E}-03$ & $6.684559 \mathrm{E}-03$ & $6.795211 \mathrm{E}-03$ \\
0.01 & $1.534928 \mathrm{E}-03$ & $1.582329 \mathrm{E}-03$ & $1.792941 \mathrm{E}-03$ & $2.053374 \mathrm{E}-03$ \\
0.1 & $1.239903 \mathrm{E}-03$ & $1.289946 \mathrm{E}-03$ & $1.512459 \mathrm{E}-03$ & $1.790687 \mathrm{E}-03$ \\
1.0 & $1.234630 \mathrm{E}-03$ & $1.284887 \mathrm{E}-03$ & $1.508174 \mathrm{E}-03$ & $1.787092 \mathrm{E}-03$ \\
10.0 & $1.234426 \mathrm{E}-03$ & $1.284691 \mathrm{E}-03$ & $1.508008 \mathrm{E}-03$ & $1.786954 \mathrm{E}-03$ \\
100.0 & $1.234408 \mathrm{E}-03$ & $1.284674 \mathrm{E}-03$ & $1.507994 \mathrm{E}-03$ & $1.786942 \mathrm{E}-03$ \\
\hline
\end{tabular}

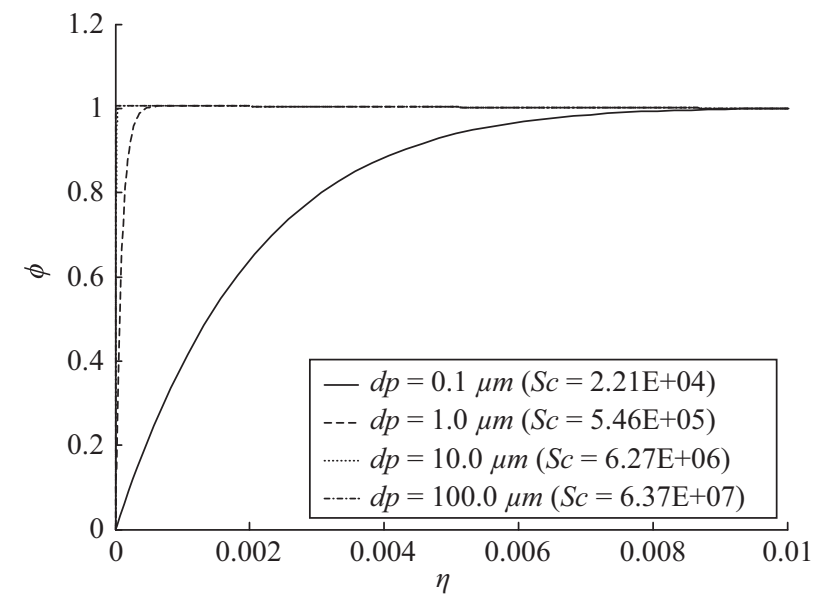

Fig. 3. Concentration profiles on cold wall surface at different particle sizes for $f_{w}=0.0, N_{t}=0.05, M=1.0, C=1.0$, and $B=0.5$.

The selected concentration profiles for different particle sizes in a range of $d p=0.1-100 \mu \mathrm{m}$, which corresponds to the value of particle diffusivity $(D)$ from $6.85 \mathrm{E}-10$ to $2.37 \mathrm{E}-13$ $\mathrm{m}^{2} / \mathrm{s}$ and Schmidt number $(S c)$ from $2.21 \mathrm{E}+04$ to $6.37 \mathrm{E}+07$ [24] are presented in Fig. 3. The larger particle size coincides with the larger particle Schmidt number. As the air kinematic viscosity is maintained, the larger particle size has weaker particle diffusivity. The weaker diffusivity leads particle to deposit on the surface accompanies the thinner concentration boundary layer. In other words, the particles of larger size deposit on the plate whereas lighter particles are drifted away from the surface.

The concentration profiles for particle size $d p=1.0 \mu \mathrm{m}$ under cold versus hot wall surfaces are shown in Figs. 4 and 5, respectively. We selected the representative thermophoretic parameter, $0 \leq N_{t} \leq 0.05$ which corresponds to the temperature difference, $\Delta T$ from $0 K$ to $30 \mathrm{~K}$ as ambient temperature, $T_{\infty}=$ $300 K$ and thermophoretic coefficient, $\kappa=0.5$ to examine the effect of thermophoresis on particle deposition onto a stretching permeable surface. Positive $N_{t}$ indicates a cold surface, whereas negative means a hot one. Fig. 4 displays that the concentration profile rises steeply at $\eta \rightarrow 0$, indicating a larger particle deposition flux, and the slope of profile increases with increasing $N_{t}$ due to the thermophoresis plays a suction-like effect on particles for a cold surface. However, under the hot wall surface condition, the thermophoretic effect leads to less particle flux to the wall and the particles might be blown away from the surface, as indicated that a zone free of particles may occur for $N_{t}=-0.05$, which is extended to about $0.01<\eta<$ 0.02 as shown in Fig. 5. 


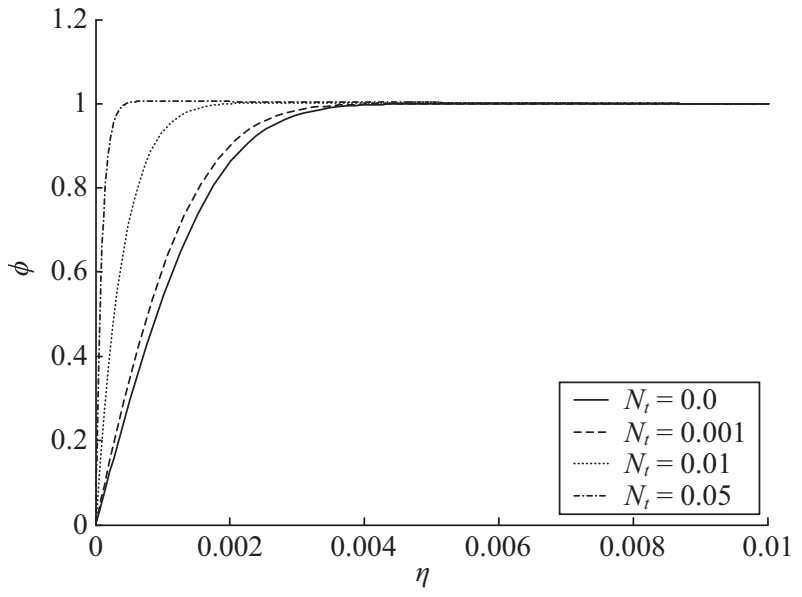

Fig. 4. Concentration profiles on cold wall surface at different $N_{t}$ for $d p=$ $1.0 \mu m, f_{w}=0.0, M=1.0, C=1.0$, and $B=0.5$.

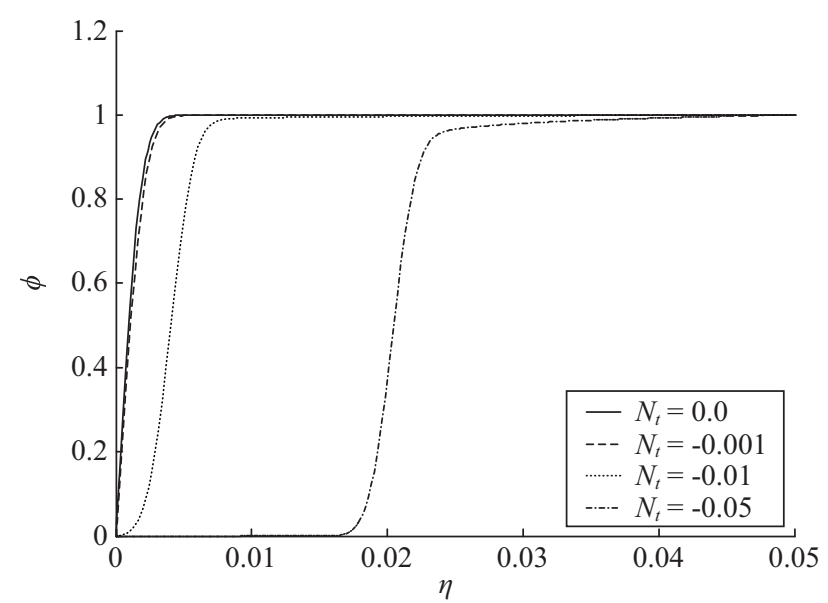

Fig. 5. Concentration profiles on hot wall surface at different $N_{t}$ for $d p=$ $1.0 \mu m, f_{w}=0.0, M=1.0, C=1.0$, and $B=0.5$.

Fig. 6 describes the calculated deposition velocities for particle size of $d p=0.001-100 \mu \mathrm{m}$ at different $N_{t}$. From the figure, it can be found that the deposition velocity increases with increasing $N_{t}$ and especially when the particle size $d p \geq$ $0.01 \mu \mathrm{m}$, thermophoresis plays an important role than the Brownian diffusion. Fig. 7 depicts the comparisons of the particle deposition velocities at different surface stretching coefficient $c=1,10$, and 100. It can be seen that the deposition velocity increases with increasing $c$ due to a larger $c$ coincides with a stronger surface stretching intensity, and the induced flow velocity is helpful for particles moving toward the surface. Fig. 8 focuses on the calculated deposition velocities considering the heat generative/absorptive effect at different source parameters $B=-0.5,0.0$, and 0.5 . For smaller particle size $d p \leq 0.1 \mu m$, the deposition velocities are primarily dominated by Brownian diffusion. Whereas, the deposition velocities decrease with increasing $B$ for larger particles $d p=0.1 \mu m$ and greater effect as the particle size becomes larger. The larger $C$ means the stronger free stream strength $a$

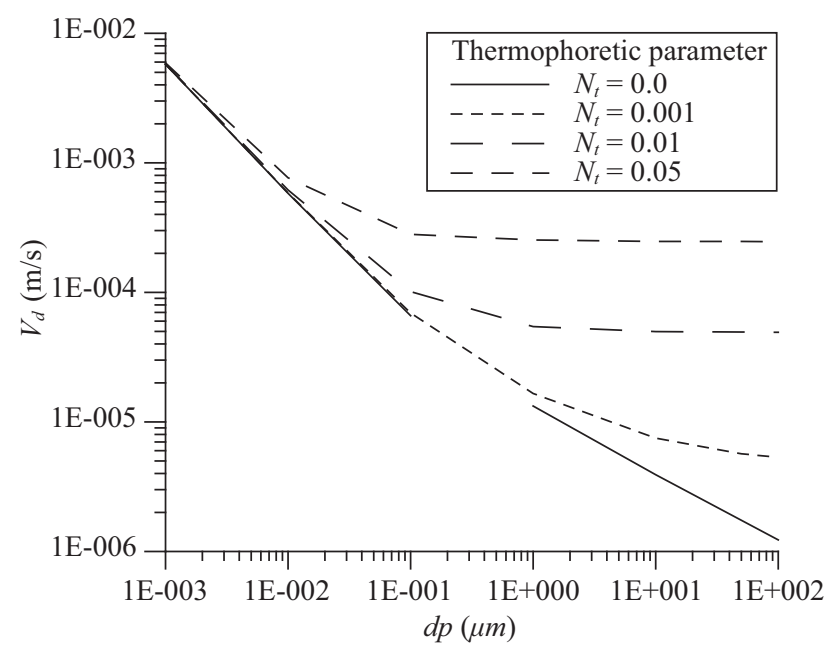

Fig. 6. Thermophoretic effect on particle deposition onto a cold wall surface at $c=10$ for $f_{w}=0.0, M=1.0, C=1.0$, and $B=0.5$.

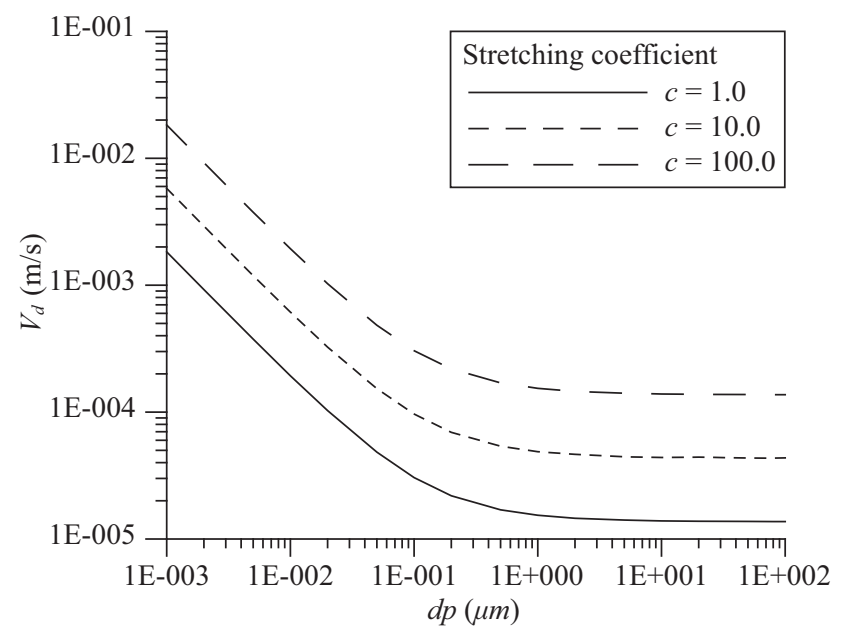

Fig. 7. Stretching effect on particle deposition onto a cold wall surface for $f_{w}=0.0, N_{t}=0.01, M=1.0, C=1.0$, and $B=0.5$.

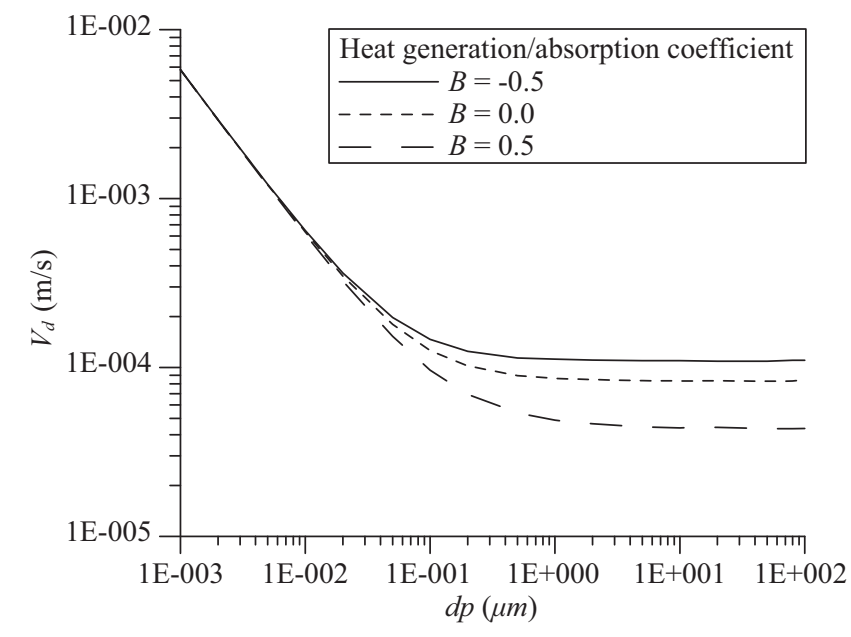

Fig. 8. Heat source effect on particle deposition onto a cold wall surface at $c=10$ for $f_{w}=0.0, N_{t}=0.01, M=1.0$, and $C=1.0$. 


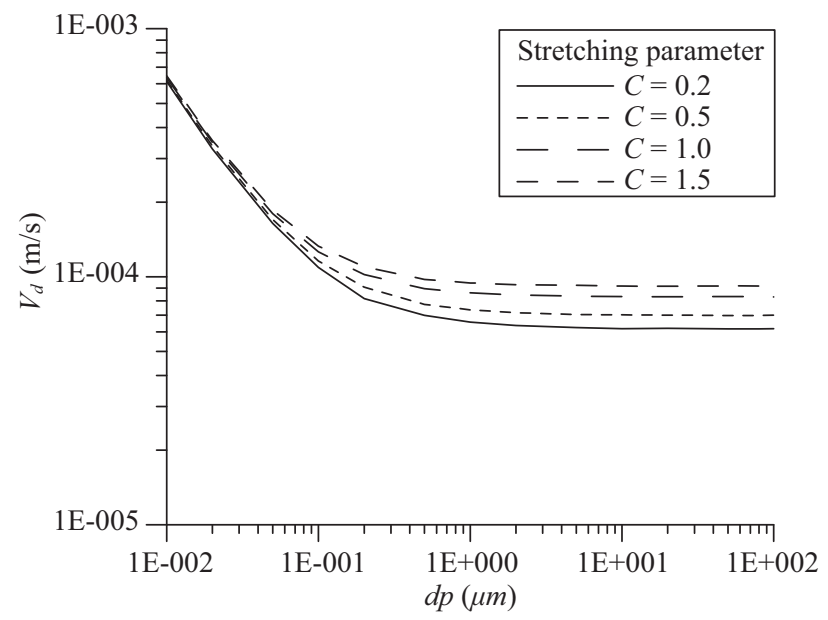

Fig. 9. Stretching parameter effect on particle deposition onto a cold wall surface at $c=10$ for $f_{w}=0.0, N_{t}=0.01, M=0.0$, and $B=0.1$.

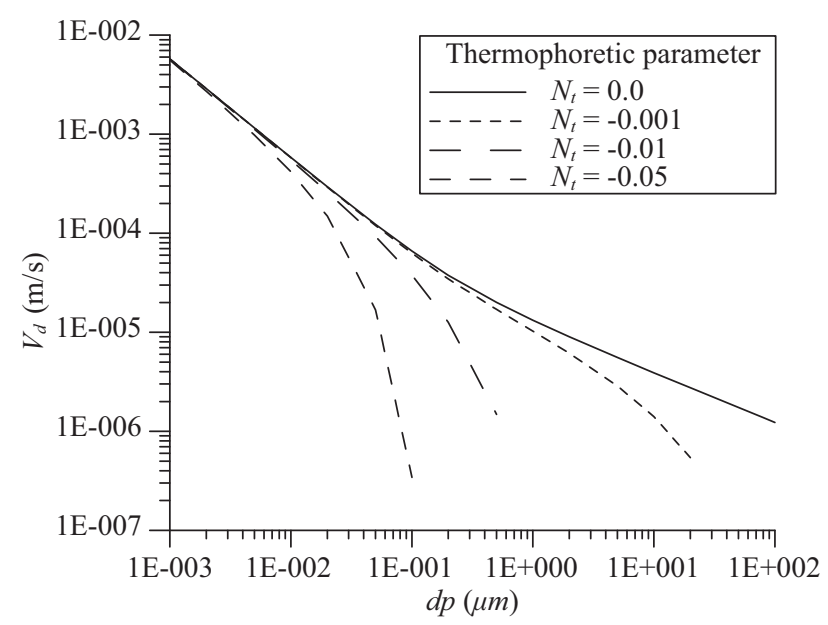

Fig. 10. Thermophoretic effect on particle deposition onto a hot wall surface at $c=10$ for $f_{w}=0.0, M=1.0, C=1.0$, and $B=0.5$.

under a constant, $c=10$. This speed up the particle deposition velocity for almost whole range of particle sizes as displayed in Fig. 9.

Fig. 10 illustrates the deposition velocities influenced by the thermophoresis that indicates a blowing-like effect driven the particles away from a hot wall under the presence of heat source. The largest temperature difference of $N_{t}=-0.05$ accompanies the temperature gradient obviously, and thus decreases the deposition velocity rapidly for $d_{p} \geq 0.1 \mu \mathrm{m}$. Fig. 11 reveals that heat source affects the particle deposition velocity which increases with the heat generation, $B>0$, but decrease with absorption, $B<0$. This is because of the heat generative or absorptive effect would lead to different temperature gradient as well as particle mobility.

\section{CONCLUSIONS}

Aerosol particles flow through a porous medium onto a stretching surface with internal heat source and wall suction/

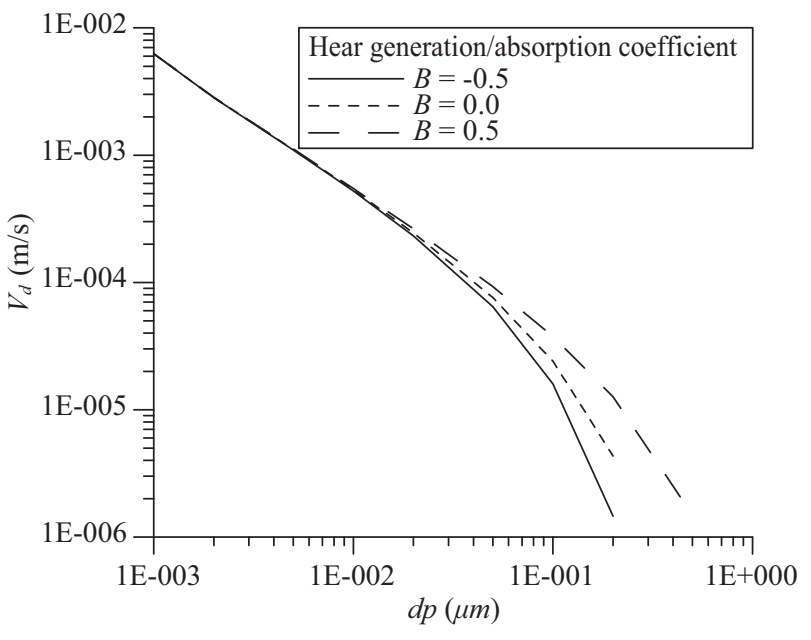

Fig. 11. Heat source effect on particle deposition onto a hot wall surface at $c=10$ for $f_{w}=0.0, N_{t}=-\mathbf{0 . 0 1}, M=1.0$, and $C=1.0$.

injection velocity is analyzed. The air-flow is modeled as a two-dimensional, steady state and incompressible flow. Similarity analysis and numerical method are performed to determine the velocity, temperature and particle concentration fields. The influences of the porosity, heat source, stretching surface and thermophoresis on the particle deposition velocities are examined. Thermophoresis for a cold surface produces a suction-like effect on particles, and a blowing-like effect for a hot one. Even if the temperature difference between the wall and the free stream is small, e.g. $N_{t}= \pm 0.01$ $(\Delta T \approx 6 K)$, thermophoresis still plays a vital role for particle size $d_{p} \geq 0.1 \mu \mathrm{m}$. According to the obtained results, we can conclude that the deposition velocity increases with the increasing thermophoretic and stretching parameters, whereas decreases with the increasing heat source parameter for a cold surface. On the other hand, the deposition velocity decreases sharply for a hot surface as the thermophoretic parameter increases but heat source parameter decreases. Through the establishment of theoretical model and numerical analysis, the particle concentration profiles and deposition rates are obtained to enhance the particle control technology in a porous medium and remove the contaminant from the air.

\section{NOMENCLATURE}

$a$ $B$

free stream strength heat source parameter stretching parameter

$C$
$C_{c}$ $C_{c} \quad$ Cunningham correction factor $C_{s} C_{t} C_{m} \quad$ constants in Eq. (6) $c$ $c_{p}$ $D$ characteristic stretching coefficient specific heat at constant pressure particle diffusivity particle diameter dimensionless flow stream function 


$\begin{array}{ll}J & \text { particle deposition flux } \\ K & \text { Darcy permeability } \\ K n & \text { Knudsen number } \\ k & \text { thermal conductivity } \\ M & \text { porosity parameter } \\ N & \text { particle concentration } \\ N_{t} & \text { thermophoretic parameter } \\ \mathrm{Pr} & \text { Prandtl number } \\ Q & \text { volumetric rate of heat generation/absorption } \\ S c & \text { Schmidt number } \\ T & \text { temperature } \\ (U, V) & \text { velocity components for the potential flow } \\ (u, v) & \text { components for the potential flow velocity at } \\ & \text { any point }(x, y) \\ V_{d} & \text { particle deposition velocity } \\ V_{T} & \text { thermophoretic velocity } \\ (x, y) & \text { streamwise and normal coordinates, respec- } \\ & \text { tively }\end{array}$

\section{Greek Symbols}

\begin{tabular}{ll}
\hline$\alpha$ & thermal diffusivity \\
$\eta$ & similarity parameter \\
$\kappa$ & thermophoretic coefficient \\
$\lambda$ & mean free path of air molecule \\
$\lambda_{g}, \lambda_{p}$ & thermal conductivities of air and particle, re- \\
$\mu$ & spectively \\
$v$ & air dynamic viscosity \\
$\rho$ & air kinematic viscosity \\
$\theta$ & air density \\
$\varphi$ & dimensionless temperature \\
$\psi$ & dimensionless particle concentration \\
$\Delta$ & stream function
\end{tabular}

\begin{tabular}{ll}
\multicolumn{2}{l}{ Subscripts and Superscripts } \\
$\infty$ & free stream \\
$w$ & wall
\end{tabular}

\section{ACKNOWLEDGMENTS}

The authors are grateful to the National Science Council, Taiwan, ROC for support through grant No. NSC 98-2221-E033-055.

\section{REFERENCES}

1. Alam, M. S., Rahman, M. M., and Sattar, M. A., "Effects of variable suction and thermophoresis on steady MHD combined free-forced convective heat and mass transfer flow over a semi-infinite permeable inclined plate in the presence of thermal radiation," International Journal of Thermal Sciences, Vol. 47, pp. 758-765 (2008).

2. Ali, M. E., "On thermal boundary layer on a power-law stretched surface with suction or injection," International Journal of Heat and Fluid Flow, Vol. 16, pp. 280-290 (1995).

3. Attia, H. A., "On the effectiveness of porosity on stagnation point flow towards a stretching surface with heat generation," Computational Materials Science, Vol. 38, pp. 741-745 (2007).
4. Banks, W. H. H., "Similarity solutions of the boundary-layer equations for a stretching wall," Journal de Mécanique Théorique et Appliquée, Vol 2, pp. 375-392 (1983).

5. Batchelor, G. K. and Shen, C., "Thermophoretic deposition in gas flow over cold surfaces," Journal of Colloid and Interface Science, Vol. 107, pp. 21-37 (1985).

6. Chamkha, A. J., Al-Mudhaf, A. F., and Pop, I., "Effect of heat generation or absorption on thermophoretic free convection boundary layer from a vertical flat plate embedded in a porous medium," International Communications in Heat and Mass Transfer, Vol. 33, pp. 1096-1102 (2006).

7. Chamkha, A. J. and Pop, I., "Effect of thermophoresis particle deposition in free convection boundary layer from a vertical flat plate embedded in a porous medium," International Communications in Heat and Mass Transfer, Vol. 31, pp. 421-430 (2004).

8. Chang, Y. P., Tsai, R., and Sui, F. M., "The effect of thermophoresis on particle deposition from a mixed convection flow onto a vertical flat plate," Journal of Aerosol Science, Vol. 30, pp. 1363-1378 (1999).

9. Elbashbeshy, E. M. A., "Heat transfer over a stretching surface with variable heat flux," Journal of Physics D: Applied Physics, Vol. 31, pp. 1951-1955 (1998).

10. Elbashbeshy, E. M. A. and Bazid, M. A. A., "Heat transfer in a porous medium over a stretching surface with internal heat generation and suction or injection," Applied Mathematics and Computation, Vol. 158, pp. 799-807 (2004).

11. Goldstein, S., Modern Developments in Fluid Dynamics, Clarendon Press, Oxford (1938).

12. Goren, S. L., "Thermophoresis of aerosol particles in the laminar boundary layer on a flat surface," Journal of Colloid and Interface Science, Vol. 61, pp. 77-85 (1977).

13. Hiemenz, K., "Die Grenzschicht an einem in den gleichformingen flussigkeitsstrom eingetauchten geraden Kreiszylinder," Dingler's Polytechnisches Journal, Vol. 326, pp. 321-410 (1911).

14. Homann, F., "Der Einfluss grosser Zahighkeit bei der Stromung um den Zylinder und um die Kugel," Zeitschrift für Angewandte Mathematik und Mechanik, Vol. 16, pp. 153-164 (1936).

15. Homsy, G. M., Geyling, F. T., and Walker, K. L., "Blasius series for thermophoresis deposition of small particles," Journal of Colloid and Interface Science, Vol. 83, pp. 495-501 (1981).

16. Mahdy, A. and Hady, F. M., "Effect of thermophoretic particle deposition in non-Newtonian free convection flow over a vertical plate with magnetic field effect," Journal of Non-Newtonian Fluid Mechanics, Vol. 161, pp. 37-41 (2009).

17. Mills, A. F., Heat and Mass Transfer, Irwin, New York (1995).

18. Nazaroff, W. W. and Cass, G. R., "Particle deposition from a natural convection flow onto a vertical isothermal flat plate," Journal of Aerosol Science, Vol. 18, pp. 445-455 (1987).

19. Opiolka, S., Schmidt, S., and Fissan, H., "Combined effects of electrophoresis and thermophoresis on particle deposition onto flat plates," Journal of Aerosol Science, Vol. 25, pp. 665-671 (1994).

20. Partha, M. K., "Suction/injection effects on thermophoresis particle deposition in a non-Darcy porous medium under the influence of Soret, Dufour effects," International Journal of Heat and Mass Transfer, Vol. 52, pp. 1971-1979 (2009).

21. Peters, M. H. and Cooper, D. W., "The effects of electrostatic forces on thermophoretic suppression of particle diffusional deposition onto hot surfaces," Journal of Colloid and Interface Science, Vol. 140, pp. 48-56 (1990).

22. Postelnicu, A., "Effects of thermophoresis particle deposition in free convection boundary layer from a horizontal flat plate embedded in a porous medium," International Journal of Heat and Mass Transfer, Vol. 50, pp. 2981-2985 (2007).

23. Rashad, A. M., "Influence of radiation on MHD free convection from a vertical flat plate embedded in porous media with thermophoretic deposition of particles," Communications in Nonlinear Science and Numerical Simulation, Vol. 13, pp. 2213-2222 (2008).

24. Reist, P. C., Aerosol Science and Technology, second ed., MacGraw-Hill, 
New York (1993).

25. Sakiadis, B. C., "Boundary layer behavior on continuous solid surfaces: I. Boundary layer equations for two-dimensional and axisymmetric flow," AIChE Journal, Vol. 7, pp. 26-28 (1961).

26. Seddeek, M. A., "Influence of viscous dissipation and thermophoresis on Darcy-Forcheimer mixed convection in a fluid saturated porous media," Journal of Colloid and Interface Science, Vol. 293, pp. 137-142 (2006).

27. Selim, A., Hossain, M. A., and Rees, D. A. S., "The effect of surface mass transfer on mixed convection flow past a heated vertical flat permeable plate with thermophoresis," International Journal of Thermal Sciences, Vol. 42, pp. 973-982 (2003).

28. Sibulkin, M., "Heat transfer near the forward stagnation point of a body of revolution," Journal of Aeronautical Sciences, Vol. 19, pp. 570-571 (1952).

29. Talbot, L., Cheng, R. K., Scheffer, R. W., and Wills, D. P., "Thermopho- resis of particles in a heated boundary layer," Journal of Fluid Mechanics, Vol. 101, pp. 737-758 (1980).

30. Tsai, R., "A simple approach for evaluating the effect of wall suction and thermophoresis on aerosol particle deposition from a laminar flow over a flat plate," International Communications in Heat and Mass Transfer, Vol. 26, pp. 249-257 (1999).

31. Tsai, R., Chang, Y. P., and Lin, T. Y., "Combined effects of thermophoresis and electrophoresis on particle deposition onto a wafer," Journal of Aerosol Science, Vol. 29, pp. 811-825 (1998).

32. Tsai, R. and Huang, J. S., "Heat and mass transfer for Soret and Dufour's effects on Hiemenz flow through porous medium onto a stretching surface," International Journal of Heat and Mass Transfer, Vol. 52, pp. 2399-2406 (2009).

33. White, F. M., Viscous Fluid Flow, second ed., MacGraw-Hill, New York (1991). 